\title{
Flute instability and the associated radial transport in the tandem mirror with a divertor mirror cell
}

\author{
I. Katanuma, ${ }^{\text {a) }}$ K. Yagi, Y. Haraguchi, N. Ichioka, S. Masaki, M. Ichimura, and T. Imai \\ Plasma Research Center, University of Tsukuba, Tsukuba, Ibaraki 305-8577, Japan
}

(Received 25 August 2010; accepted 29 September 2010; published online 11 November 2010)

\begin{abstract}
The flute instability and the associated radial transport are investigated in the tandem mirror with a divertor mirror cell (the GAMMA10 A-divertor) with help of computer simulation, where GAMMA10 is introduced [Inutake et al., Phys. Rev. Lett. 55, 939 (1985)]. The basic equations used in the simulation were derived on the assumption of an axisymmetric magnetic field. So the high plasma pressure in a nonaxisymmetric minimum-B anchor mirror cell, which is important for the flute mode stability, is taken into account by redefining the specific volume of a magnetic field line. It is found that the flute modes are stabilized by the minimum-B magnetic field even with a divertor mirror although its stabilizing effects are weaker than that without the divertor mirror. The flute instability enhances the radial transport by intermittently repeating the growing up and down of the Fourier amplitude of the flute instability in time. (c) 2010 American Institute of Physics.

[doi:10.1063/1.3503772]
\end{abstract}

\section{INTRODUCTION}

The GAMMA10 tandem mirror ${ }^{1-3}$ is planning to replace one of anchor cells with an axisymmetric divertor mirror cell (called GAMMA10 A-divertor), which is illustrated in Fig. 1. A role of the GAMMA10 A-divertor is to evacuate the ions rapidly through a magnetic null point ( $\mathrm{x}$-point) to the dipole region outside the divertor mirror cell. A divertor plate is installed in the dipole region in order to catch the ions lost from the divertor region, which is anticipated being the simulation experiment of the divertor of a big torus. ${ }^{4-7}$

The $\mathrm{x}$-point has the following roles. Unmagnetized electrons can move freely along a magnetic null line azimuthally, so that the electrostatic potential is the same on the magnetic flux tube passing through x-point (separatrix). Thus the electrostatic fluctuations are stabilized by the short circuit effects on the separatrix. Ions diffused to the separatrix in the central cell, therefore, move along a magnetic field line and finally escape through $\mathrm{x}$-point to the dipole region. The magnetic null has a role of hole on the separatrix, where ions on the separatrix can move freely to the dipole region due to the nonconservation of ion magnetic moment.

The GAMMA10 A-divertor has two experimental operation modes. One is the steady state operation, where ions diffused radially in the central cell move along a separatrix to $\mathrm{x}$-point and then are lost to the divertor plate continuously. The flute mode fluctuations can cause a large ion radial diffusion in the steady state. ${ }^{8}$ The nonaxisymmetric electrostatic potential in the central cell, which is formed artificially by the electron cyclotron resonance heating, can also enhance the large ion radial transport ${ }^{9}$ as well as the neoclassical transport due to the nonaxisymmetric magnetic field. ${ }^{10-13}$

Another operation is the one like a disruption observed in a big torus ${ }^{14,15}$ or edge localized mode observed in a divertor tokamak, ${ }^{16}$ where the plasma is made lost rapidly from the central cell. The rapid loss of plasma is realized with help

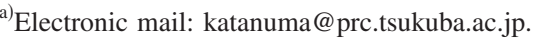

of flute instabilities, which is one of the purpose of this paper.

The flute modes in the GAMMA10 A-divertor operation are stabilized by the high plasma pressure created in a remaining nonaxisymmetric anchor mirror cell. ${ }^{17}$ Thus the flute modes are destabilized when the high plasma pressure is destroyed by gas puffing in the anchor mirror cell. Another purpose of this paper is to make clear the stabilizing effects by a minimum-B mirror in the tandem mirror with a divertor mirror cell.

\section{FLUTE STABILITY}

The flute modes are able to be stabilized in an axisymmetric divertor mirror by means of plasma compressibility, if $p U^{\gamma}=$ const is realized radially. ${ }^{18}$ Here $p$ is plasma pressure, $U$ is the specific volume of a magnetic field line defined by $U \equiv \int \mathrm{d} \ell / B$, where $B$ is the magnetic field and the integration is carried out along a magnetic field line, and $\gamma$ is the specific heat index; $\gamma=5 / 3$ is assumed through this paper. Pressure $p$ can be zero at the separatrix if $p U^{\gamma}=$ const is satisfied because $U$ of the magnetic field line passing through $\mathrm{x}$-point is infinitely large. The stability analysis of the divertor mirror, taking into account the effects of the ion finite Larmor radius, magnetic field line curvature, and the plasma compressibility, has been done. ${ }^{19-21}$ It was reported that the flute modes were stabilized by the divertor mirror experimentally. $^{22-24}$

Because the classical radial transport is large around $\mathrm{x}$-point, the diffusion forms locally stable pressure profile (with $\partial U / \partial \psi$ ) in the neighborhood of magnetic null and unstable pressure profile outside this area and so $\partial p U^{\gamma} / \partial \psi=0$ is not satisfied around the separatrix (i.e., around the plasma boundary), which can destabilize the flute modes. The quantity $\psi$ relates to the magnetic flux defined by $\boldsymbol{B}=\nabla \psi \times \nabla \varphi$, where $(\psi, \varphi, \ell)$ coordinates are used. We consider the flute mode stability criterion 


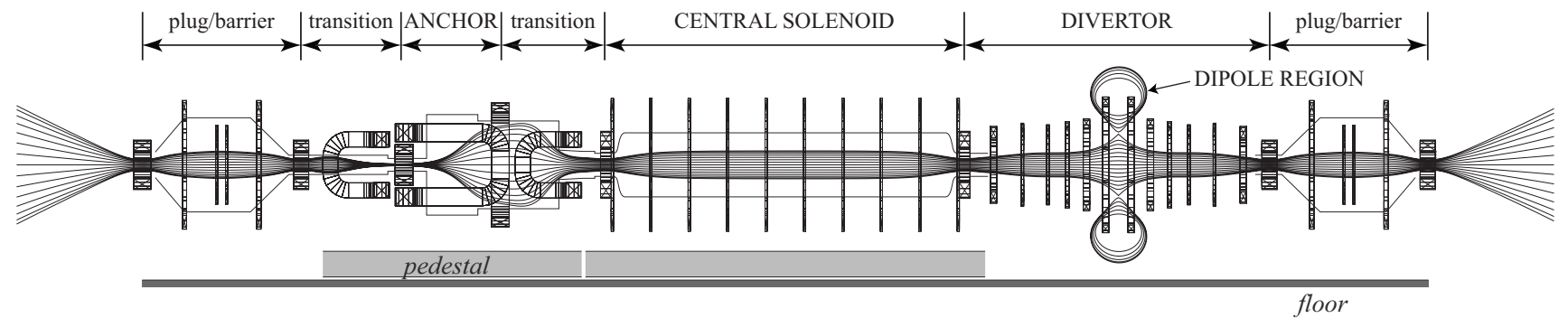

FIG. 1. Schematic diagram of the GAMMA10 A-divertor. The magnetic field lines are plotted with coils and the GAMMA10 vacuum vessel.

$$
\int \frac{\left(\hat{p}_{\perp}+\hat{p}_{\|}\right) \kappa_{\psi}}{B} d \ell \geq 0
$$

Here the normal curvature $\kappa_{\psi}$ is defined by a magnetic field line curvature $\boldsymbol{\kappa} \equiv \hat{e}_{\|} \cdot \nabla \hat{e}_{\|}=\kappa_{\psi} \nabla \psi+\kappa_{\varphi} \nabla \varphi$, where $\hat{e}_{\|} \equiv \boldsymbol{B} / B$ and plasma pressures $\hat{p}_{\|, \perp}$ are represented by a separation of variables $p_{\|, \perp}(\psi, B)=\hat{p}_{\|, \perp}(B) \nu(\psi)$. Although Eq. (1) was derived in the long thin approximation in Refs. 17, 25, and 26, the stability criterion (1) can be applied to the fat mirror plasma. $^{27,28}$

Equation (1) is rewritten as

$$
\int \frac{\left(\hat{p}_{\perp}+\hat{p}_{\|}\right) \kappa_{\psi}}{B} d \ell=-\frac{1}{2} \frac{\partial}{\partial \psi} \int \frac{\left(\hat{p}_{\perp}+\hat{p}_{\|}\right)}{B} d \ell
$$

in the vacuum magnetic field because of $\nabla_{\perp} B(\psi, \varphi, \ell)=B \boldsymbol{\kappa}$. Note that Eqs. (1) and (2) are applicable to a divertor fat plasma. $^{27,28}$ The stability criterion (1), therefore, reduces to the classical stability criterion of flute modes

$$
\partial U / \partial \psi \leq 0
$$

in the mirror cell, ${ }^{29}$ if the specific volume of a magnetic field line $U$ is redefined as

$$
U \equiv \int \frac{\left(\hat{p}_{\perp}+\hat{p}_{\|}\right)}{B} d \ell .
$$

Equation (4) takes into account the anisotropic pressure effects in the stability criterion (3) of the divertor mirror cell, and Eq. (3) is able to be applied to the effectively axisymmetrized tandem mirror such as the GAMMA10 A-divertor.

Strictly speaking, the $\psi$ derivative on the right-hand side of Eq. (2) acts on $\left(\hat{p}_{\perp}+\hat{p}_{\|}\right)$as well as $1 / B$, i.e.

$$
\begin{aligned}
\frac{\partial}{\partial \psi} \int \frac{\left(\hat{p}_{\perp}+\hat{p}_{\|}\right)}{B} d \ell= & \int\left\{\left(\frac{\partial}{\partial \psi} \frac{1}{B^{2}}\right)\left(\hat{p}_{\perp}+\hat{p}_{\|}\right)\right. \\
& \left.+\frac{1}{B^{2}}\left[\frac{\partial}{\partial \psi}\left(\hat{p}_{\perp}+\hat{p}_{\|}\right)\right]\right\} B d \ell .
\end{aligned}
$$

Equation (2), therefore, is correct on condition that $\left|\left(\hat{p}_{\perp}+\hat{p}_{\|}\right)^{-1} \partial\left(\hat{p}_{\perp}+\hat{p}_{\|}\right) / \partial \psi\right| \ll\left|B^{2} \partial B^{-2} / \partial \psi\right|$. We expect that this condition is satisfied in the long thin approximation, and then in the anchor mirror cell.

The axial pressure profiles used in this paper are plotted in Fig. 2, which are given by

$$
\hat{p}(B) \equiv \hat{p}_{\perp}(B)+\hat{p}_{\|}(B)=\max \left[p_{A} \frac{\left(B_{m}^{2}-B^{2}\right)}{\left(B_{m}^{2}-B_{c}^{2}\right)}, 1\right] .
$$

Here $p_{A}$ is the pressure at the anchor midplane, $B_{c}$ is the magnetic field at the midplane on axis in anchor cell, and $B_{m}=1.7 B_{c}$. The pressure $\hat{p}$ in the other region is assumed to be unity.

The radial profiles of $U$ for various anchor pressure $p_{A}$ are plotted as a function of $x$ in Fig. 3. Here $x$ is the normalized radial coordinate defined by $x=\sqrt{\psi / \psi_{b}}$, where $\psi_{b}$ is the coordinate at the separatrix. Because the GAMMA10 A-divertor is an effectively axisymmetrized tandem mirror, $U$ is a function of only $\psi$ but not $\varphi$. An appearance of $\partial U / \partial x<0$ around $x \simeq 0$ in the case of $p_{A} \geq 12$ in Fig. 3 means that the flute modes are locally stable in the core region. The nonlocal analysis, however, is required to determine the linear growth rate of a flute instability, the detail of which is given in Sec. IV because the flute instability is a global mode.

The radial profiles of the normalized dynamic vorticity $w_{0}(x)$, density $D_{E}(x)$, and temperature $T_{E}(x)$ are assumed to be

$$
w_{0}(x)=+1.0, \quad D_{E}(x)=1.0
$$

and

$$
T_{E}(x)=\exp \left\{-2 x^{2}\right\}[U(x) / U(0)]^{\gamma-1}
$$

in the calculation of linear growth rate. The definitions of $w_{0}$, $D_{E}$, and $T_{E}$ are given in Sec. III.

Figure 4 plots the linear growth rates of $m=1$ flute mode, where $m$ is the azimuthal mode number. Flute modes are stable for all $U \mathrm{~s}$ in Fig. 3, if $T_{E}(x)=1.0$. The linear growth rates of flute instability in the case of a slim $T_{E}(x)$ of Eq. (7)

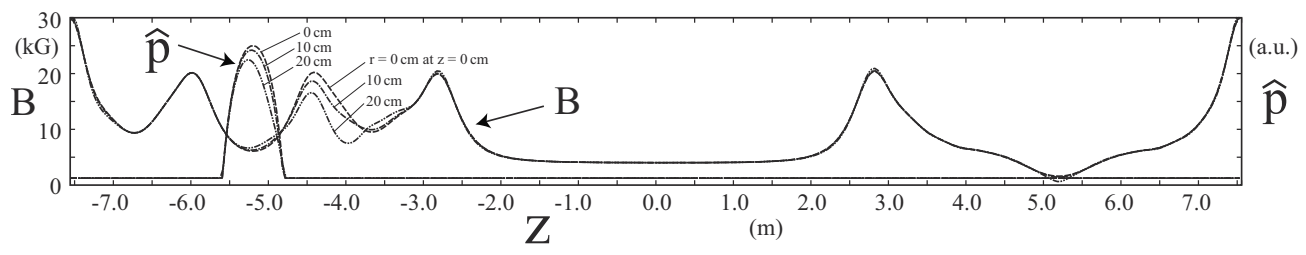

FIG. 2. Axial profiles of pressure $\hat{p}=\hat{p}_{\|}+\hat{p}_{\perp}$ in the GAMMA10 A-divertor. 


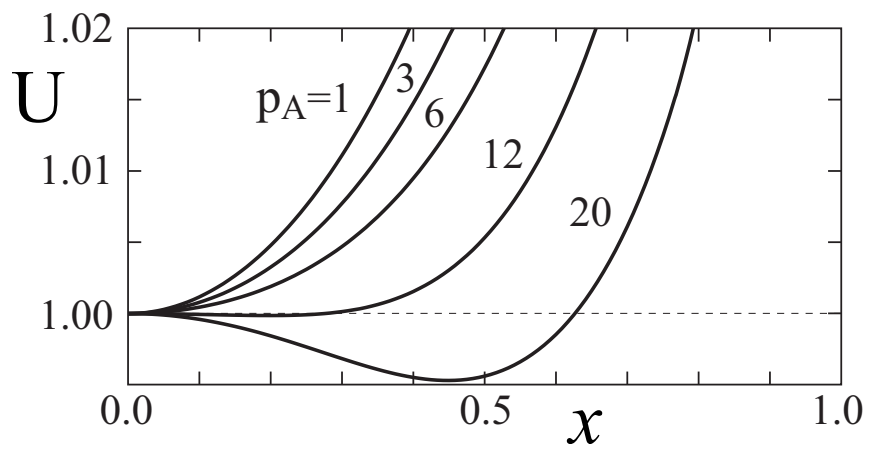

FIG. 3. The radial profiles of the normalized specific volume $u(x)=U(x) / U(0)$ for various $p_{A}$.

are plotted by solid circles and in a fatter $T_{E}(x)$ case of Eq. (8) are plotted by solid triangles in Fig. 4, where

$$
T_{E}(x)=\exp \left\{-0.5 x^{2}\right\}[U(x) / U(0)]^{\gamma-1} .
$$

It is found that the linear growth rates of the flute mode are larger in the slim temperature than those in the fatter temperature. In the long thin approximation, ${ }^{17,25,26}$ the flute modes are understood to be stable as long as $\partial U(x) / \partial x \leq 0$ at $x \simeq 0$. Figure 4 , however, reveals that the flute modes are not stable even if $\partial U(x) / \partial x \leq 0$ at $x \simeq 0$ (i.e., $p_{A} \geq 12$ in Fig. 3). The stability boundary of a flute instability can be changed by the temperature and density radial profiles. ${ }^{21}$ Thus the self-consistent numerical simulation is necessary to estimate the flute instability in a divertor mirror.

\section{BASIC EQUATIONS}

In this section we describe the basic equations used in the simulation, which are in the framework of magnetohydrodynamic (MHD) equations, by which the nonlinear MHD convection and the flute mode fluctuations are studied. The detailed derivation of the following basic equation is described in Refs. 8, 30, and 31. The normalized specific dynamic vorticity $\hat{w}$, normalized mass density $\hat{D}$, and normalized effective temperature $\hat{T}$, which are the quantities integrated over a unit magnetic flux tube, are defined as

$$
\begin{aligned}
\hat{w}(x, \varphi) \equiv & \frac{\partial}{2 x \partial x}\left(\hat{D}\left\langle r^{2} / b^{2}\right\rangle \frac{\partial \phi}{2 x \partial x}\right) \\
& +b^{2} B_{M}^{2} \frac{\partial}{\partial \varphi}\left(\hat{D}\left\langle\frac{1}{r^{2} B^{2}}+\lambda^{2} B^{2}\right\rangle \frac{\partial \phi}{\partial \varphi}\right), \\
\hat{D}(x, \varphi) \equiv & \frac{1}{\rho_{M} U_{0}} \int \frac{\rho d \ell}{B}=\frac{\rho U}{\rho_{M} U_{0}},
\end{aligned}
$$

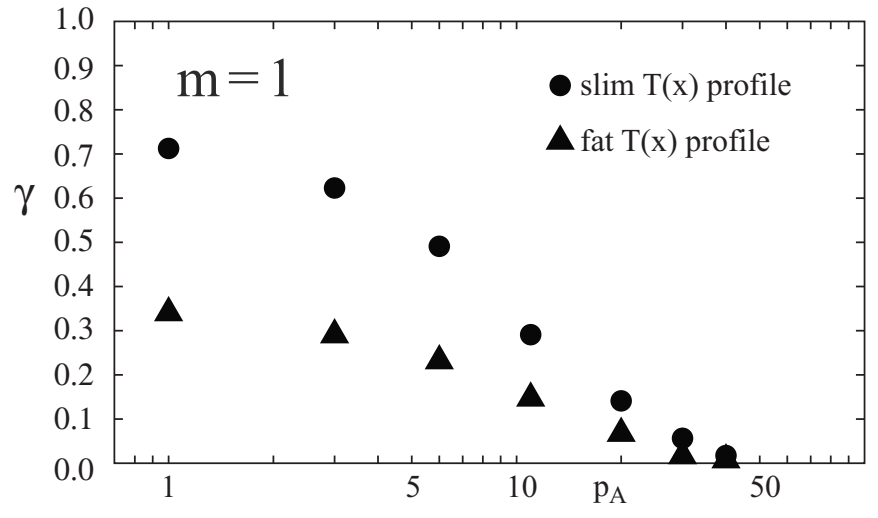

FIG. 4. Linear growth rates of the flute instability for various $p_{A}$.

$$
\hat{T}(x, \varphi) \equiv \frac{\left(T_{i}+T_{e}\right) U^{\gamma-1}}{\left(T_{i}+T_{e}\right)_{M} U_{0}^{\gamma-1}},
$$

where $\rho, T_{e}$, and $T_{i}$ are the mass density, electron, and ion temperatures, all of which are assumed to be constant along a magnetic field line; $\phi$ is the electrostatic potential multiplied by light speed $c, \quad \lambda=U(\partial / \partial \psi)\left(\int_{0}^{\ell}[1 / U J] d \ell\right)$ $+(\nabla \psi \cdot \nabla \ell) /\left(r^{2} B^{2} J\right)$ with Jacobian $J=\nabla \psi \times \nabla \varphi \cdot \nabla \ell$, and $r$ is the radius of a magnetic field line. The normalized radial coordinate $x$ is defined as $x=\sqrt{\psi / \psi_{b}}$, where $\psi_{b}$ is the coordinate at the separatrix, $b=\sqrt{\psi_{b} / B_{M}}$, and $U_{0}=U(0)$. The subscript ${ }_{M}$ means the quantity at the midplane on axis of the divertor mirror cell. The field line averaged quantity $\langle X\rangle$ is defined by $\langle X\rangle \equiv(1 / U) \int(X / B) d \ell$.

The adiabatic velocity $\boldsymbol{v}_{\alpha}$ of plasma has the form of

$$
\boldsymbol{v}_{\alpha}=\frac{-\nabla \phi \times \boldsymbol{B}}{B^{2}}+\boldsymbol{B} \lambda \frac{\partial \phi}{\partial \varphi},
$$

where the first and second terms in the right-hand side are the velocities across and along a magnetic field line, and Eq. (10) satisfies $\nabla \cdot\left(\boldsymbol{v}_{\alpha} / U\right)=0$. The specific dynamic vorticity $\hat{w}$ in Eq. (9) is written by

$$
\hat{w}(x, \varphi)=-Y_{0} \int \frac{d \ell}{J}\left\{\nabla \cdot\left[\frac{\boldsymbol{B} \times\left(\rho \boldsymbol{v}_{\alpha}\right)}{B^{2}}\right]-\frac{\partial\left[\lambda \boldsymbol{B} \cdot\left(\rho \boldsymbol{v}_{\alpha}\right)\right]}{\partial \varphi}\right\}
$$

in terms of $\boldsymbol{v}_{\alpha}$, where $Y_{0}$ is the normalization constant.

The basic equations, which have been derived on the assumption of axisymmetric magnetic field, consist of the equations of $\hat{w}(x, \varphi), \hat{D}(x, \varphi), \hat{T}(x, \varphi)$, and the potential $\Phi(x, \varphi)$ determined by Eq. (9).

Time evolutions of $\hat{w}(x, \varphi), \hat{D}(x, \varphi)$, and $\hat{T}(x, \varphi)$ are given by the equations

$$
\frac{\partial \hat{w}}{\partial \tau}+\llbracket \Phi, \hat{w} \rrbracket-\frac{1}{2} \llbracket \hat{D}, V^{2} \rrbracket+\frac{3}{10 x} \frac{1}{u^{5 / 3}} \frac{\partial u}{\partial x} \frac{\partial \hat{D} \hat{T}}{\partial \varphi}=\epsilon^{2} C_{2}\left\{\frac{1}{4 x} \frac{\partial}{\partial x}\left[\frac{D_{E} f_{1}}{x} \frac{\partial}{\partial x}\left(\frac{f_{2}}{u^{2 / 3} f_{1}^{2} \sqrt{T_{E}}} \hat{w}\right)\right]+\frac{D_{E} f_{2}\left(f_{3}+f_{4}\right)}{u^{2 / 3} f_{1}^{2} \sqrt{T_{E}}} \frac{\partial^{2} \hat{w}}{\partial \varphi^{2}}\right\}
$$




$$
\begin{aligned}
\frac{\partial \hat{D}}{\partial \tau}+\llbracket \Phi, \hat{D} \rrbracket= & \frac{\epsilon^{2} C_{1}}{4 x} \frac{\partial}{\partial x}\left[\frac{u f_{1}}{x} \frac{D_{E}}{T_{E}^{3 / 2}} \frac{\partial}{\partial x}\left(\frac{T_{E} \hat{D}}{u^{5 / 3}}\right)\right] \\
& +\epsilon^{2} C_{1} \frac{f_{3} D_{E}}{u^{2 / 3} \sqrt{T_{E}}} \frac{\partial^{2} \hat{D}}{\partial \varphi^{2}}, \\
\frac{\partial \hat{T}}{\partial \tau}+\llbracket \Phi, \hat{T} \rrbracket= & \epsilon^{2} C_{3} \frac{u^{2 / 3}}{12 x D_{E}} \frac{\partial}{\partial x}\left[\frac{D_{E}^{2} f_{1}}{x u} \frac{\partial}{\partial x}\left(\frac{\hat{T}}{u^{1 / 3} \sqrt{T_{E}}}\right)\right] \\
& +\epsilon^{2} C_{3} \frac{D_{E} f_{3}}{3 u^{2 / 3} \sqrt{T_{E}}} \frac{\partial^{2} \hat{T}}{\partial \varphi^{2}} \\
& +\frac{C_{1} \epsilon^{2}}{6 x \sqrt{T_{E}}} \frac{\partial}{\partial x}\left[\frac{u f_{1}}{x} \frac{\partial}{\partial x}\left(\frac{D_{E} T_{E}}{u^{5 / 3}}\right)\right] .
\end{aligned}
$$

Here the notation $\llbracket \rrbracket$ defined by the equation

$$
\llbracket A, B \rrbracket \equiv \frac{\partial A}{2 x \partial x} \frac{\partial B}{\partial \varphi}-\frac{\partial A}{\partial \varphi} \frac{\partial B}{2 x \partial x}
$$

is known as the Poisson bracket and this term represents the convective $\boldsymbol{E} \times \boldsymbol{B}$ flow term in Eqs. (11)-(13). The quantity $V^{2}$ in Eq. (11) is the normalized counterpart of $\left\langle v_{\alpha}^{2}\right\rangle$. The specific volume of a magnetic field line is normalized as $u(x) \equiv U(x) / U(0) ; \quad D_{E}(x) \equiv(1 / 2 \pi) \int_{0}^{2 \pi} \hat{D}(x, \varphi) d \varphi \quad$ and $T_{E}(x) \equiv(1 / 2 \pi) \int_{0}^{2 \pi} \hat{T}(x, \varphi) d \varphi$.

The potential $\Phi$ is determined by Eq. (9) as

$$
\frac{1}{x} \frac{\partial}{\partial x}\left(\frac{\hat{D} f_{1}}{x} \frac{\partial \Phi}{\partial x}\right)+4 \frac{\partial}{\partial \varphi}\left[\hat{D}\left(f_{3}+f_{4}\right) \frac{\partial \Phi}{\partial \varphi}\right]=4 \hat{w} \text {. }
$$

The constants are given by

$$
\begin{aligned}
& C_{1}=\sqrt{\frac{2 M_{e} T_{e}}{M_{i} T_{i}}}\left(\frac{T_{i}+T_{e}}{2 T_{e}}\right)_{M}^{2}, \\
& C_{2}=\frac{3}{20}\left(\frac{T_{i}+T_{e}}{T_{i}}\right)_{M}^{1 / 2}\left(\frac{T_{i}+T_{e}}{2 T_{i}}\right)_{M}, \\
& C_{3}=\left(\frac{T_{i}+T_{e}}{T_{i}}\right)_{M}^{1 / 2}, \\
& f_{1}=\left\langle r^{2}\right\rangle / b^{2}, \quad f_{2}=\left\langle r^{4}\right\rangle / b^{4}, \quad f_{3}=\left\langle\frac{1}{r^{2} B^{2}}\right\rangle B_{M}^{2} b^{2}, \\
& f_{4}=\left\langle\lambda^{2} B^{2}\right\rangle B_{M}^{2} b^{2} .
\end{aligned}
$$

The right-hand sides in Eqs. (11)-(13) are the classical transport terms related to the classical viscosity, resistivity, and thermal diffusivity. ${ }^{32}$ The quantity $\epsilon^{2}$, which is a small expansion parameter, is defined by $\epsilon^{2} \equiv\left(\chi_{M} / b c_{s M}\right)\left[2 T_{i} /\left(T_{i}\right.\right.$ $\left.\left.+T_{e}\right)\right]_{M}^{1 / 2}$, where $c_{s M}=\sqrt{\gamma p_{M} / \rho_{M}}$ and $\chi_{M}=\left[T_{i} /\left(M_{i} \omega_{c i} \tau_{i}\right)\right]_{M}$ which is related to the classical thermal diffusibility; $\epsilon^{2}=10^{-2}$ is assumed in the paper. The quantities $\omega_{c i}$ and $\tau_{i}$ are the ion cyclotron frequency and the ion coulomb collision time, respectively.

The basic equations can be applied under the assumption that the plasma pressure is below the instability threshold for Alfvén modes $\left(\beta<\beta_{c r} \sim 1, \beta\right.$ is plasma pressure/magnetic pressure), and the high frequency stable collective degrees of freedom corresponding to magnetosonic, Alfvén, and longitudinal acoustic modes are excluded from the basic equations. ${ }^{8,30,31}$ The effects of magnetic field line curvature on the flute interchange modes are included through the specific volume $u$. The basic equations do not use the long thin approximation, thus these equations can be applied to the divertor fat mirror plasma.

The specific volume $U$ in this section is defined by $U=\int d \ell / B$ and the integration along a magnetic field line is not weighted with the plasma pressure. However in order to take into account the effect of nonaxisymmetric anchor magnetic field in the basic equations, the specific volume is redefined by Eq. (4) with axial pressure profile of Eq. (5). Only the normalized specific volume $u$ appearing explicitly in Eqs. (11)-(13) uses Eq. (4).

\section{LINEAR ANALYSIS}

Equations (11)-(13) and (15) give a set of equations for the nonlinear MHD convection. The linear analysis is helpful to understanding the simulation results in Sec. V. The perturbed quantity $w_{f}$ is expanded in Fourier series as

$$
w_{f}=\sum_{m} w_{f(m)} \exp \{-i \omega \tau+i m \varphi\} .
$$

Here $\quad w_{f}(x, \varphi)=\hat{w}(x, \varphi)-w_{0}(x) \quad$ and $\quad w_{0}(x)$ $\equiv(1 / 2 \pi) \int_{0}^{2 \pi} \hat{w}(x, \varphi) \mathrm{d} \varphi ; i \equiv \sqrt{-1}, \omega$ is the frequency of linear wave, and $m$ is the azimuthal mode number. The remaining perturbed quantities $D_{f}, T_{f}$, and $\Phi$, where $D_{f}(x, \varphi)=\hat{D}(x, \varphi)$ $-D_{E}(x), T_{f}(x, \varphi)=\hat{T}(x, \varphi)-T_{E}(x)$, are expanded in the same way.

Because the quantities $\tilde{\Phi}_{(m)}, w_{f(m)}, D_{f(m)}, T_{f(m)}$, and $u$ are a function of $x$, the nonlocal treatment is required to obtain the dispersion equation, the result of which is given as

$$
\frac{\partial}{\partial x}\left[\frac{D_{E} f_{1}}{x} \frac{\partial \tilde{\Phi}_{(m)}}{\partial x}\right]-\frac{\partial}{\partial x}\left[\frac{m f_{1}}{2 x^{2}} \widetilde{\Phi}_{(m)} \frac{\partial \Phi_{0}}{\partial x} \frac{\partial D_{E}}{\partial x} /\left(\omega-\frac{m}{2 x} \frac{\partial \Phi_{0}}{\partial x}\right)\right]-\left[4 x D_{E}\left(f_{3}+f_{4}\right) m^{2}+\frac{4 x w_{f(m)}}{\widetilde{\Phi}_{(m)}}\right] \widetilde{\Phi}_{(m)}=0 .
$$

Here $\Phi_{0}$, where $\Phi_{0}(x)=(1 / 2 \pi) \int_{0}^{2 \pi} \Phi(x, \varphi) d \varphi$, is determined by

$$
\frac{\partial}{\partial x}\left(\frac{D_{E} f_{1}}{x} \frac{\partial \Phi_{0}}{\partial x}\right)=4 x w_{0} \text {. }
$$

The last term $w_{f(m)} / \widetilde{\Phi}_{(m)}$ in the left-hand side of Eq. (19) is described as a function of zeroth-order quantities ${ }^{8}$ 


$$
\begin{aligned}
\frac{4 x w_{f(m)}}{\widetilde{\Phi}_{(m)}}= & -\frac{m^{2}}{2 x} \frac{\partial\left(V^{2}\right)_{(0)}}{\partial x} \frac{\partial D_{E}}{\partial x} /\left\{\left(\omega-\frac{m}{2 x} \frac{\partial \Phi_{0}}{\partial x}\right)\left[\omega-\frac{m}{2 x} \frac{\partial \Phi_{0}}{\partial x}+\mathrm{i} m^{2} \epsilon^{2} C_{2} \frac{D_{E} f_{2}\left(f_{3}+f_{4}\right)}{u^{2 / 3} f_{1}^{2} \sqrt{T_{E}}}\right]\right\}-2 m \frac{\partial w_{0}}{\partial x} /\left[\omega-\frac{m}{2 x} \frac{\partial \Phi_{0}}{\partial x}\right. \\
& \left.+\mathrm{i} m^{2} \epsilon^{2} C_{2} \frac{D_{E} f_{2}\left(f_{3}+f_{4}\right)}{u^{2 / 3} f_{1}^{2} \sqrt{T_{E}}}\right]-\frac{3 m^{2}}{5 \epsilon^{2} x} \frac{1}{u^{5 / 3}} \frac{\partial u}{\partial x} \frac{\partial\left(D_{E} T_{E}\right)}{\partial x} /\left\{\left(\omega-\frac{m}{2 x} \frac{\partial \Phi_{0}}{\partial x}\right)\left[\omega-\frac{m}{2 x} \frac{\partial \Phi_{0}}{\partial x}+\mathrm{i} m^{2} \epsilon^{2} C_{2} \frac{D_{E} f_{2}\left(f_{3}+f_{4}\right)}{u^{2 / 3} f_{1}^{2} \sqrt{T_{E}}}\right]\right\} .
\end{aligned}
$$

Equation (19) is an eigenvalue equation with eigenfunction $\widetilde{\Phi}_{(m)}(x)$ and eigenvalue $\omega$, which can be solved with a boundary condition of $\widetilde{\Phi}_{(m)}(x)=0$ at $x=0$ and at $x=1$. The solutions of Eq. (19) are plotted in Fig. 4 for the zeroth-order quantities given in Eqs. (6)-(8). Equation (19) is used in Sec. $\mathrm{V}$ in order to compare the linear analysis with the linear phase of the simulations.

\section{SIMULATION RESULTS}

Boundary conditions adopted in the simulations are that $\partial D_{E}(0) / \partial x=\partial T_{E}(0) / \partial x=\partial w_{0}(0) / \partial x=0, \partial D_{E}(1) / \partial x$ $=\partial T_{E}(1) / \partial x=0$. The boundary condition of $w_{0}(x)$ at $x=1$ is chosen that $\int_{0}^{1} w_{0}(x) x d x$ is conserved in time. The boundary conditions for perturbed quantities are that $\partial D_{f,(2 m)}(0) / \partial x$ $=\partial T_{f,(2 m)}(0) / \partial x=\partial w_{f,(2 m)}(0) / \partial x=0, \quad D_{f,(2 m+1)}(0)=T_{f,(2 m+1)}(0)$ $=w_{f,(2 m+1)}(0)=0, \quad$ and $\quad \partial D_{f,(m)}(1) / \partial x=\partial T_{f,(m)}(1) / \partial x$ $=\partial w_{f,(m)}(1) / \partial x=0$.

Initial conditions are $\hat{D}(x, \varphi)=1, \quad \hat{T}(x, \varphi)=1$, and $\hat{w}(x, \varphi)=+1$, where these initial conditions give the marginally stable state to the flute modes as mentioned in Sec. II. Those boundary and initial conditions are adopted in all simulations in this paper. Numerical algorithm for calculating Eqs. (11)-(13) is described in Ref. 8. Time step is $\Delta \tau=5 \times 10^{-5}$ and $(x \times \varphi)=\left(121 \times 2^{6}\right)$ meshes are used in the simulation.

\section{A. The case unstable to a flute mode}

At first, the simulation in case of $p_{A}=1$ is performed, which corresponds to the case without high pressure in anchor mirror cell and so the flute modes are not stabilized by the anchor mirror because $\partial u / \partial x<0$ everywhere in Fig. 3. Although the initial radial profiles of $D_{E}(x)$ and $T_{E}(x)$ are

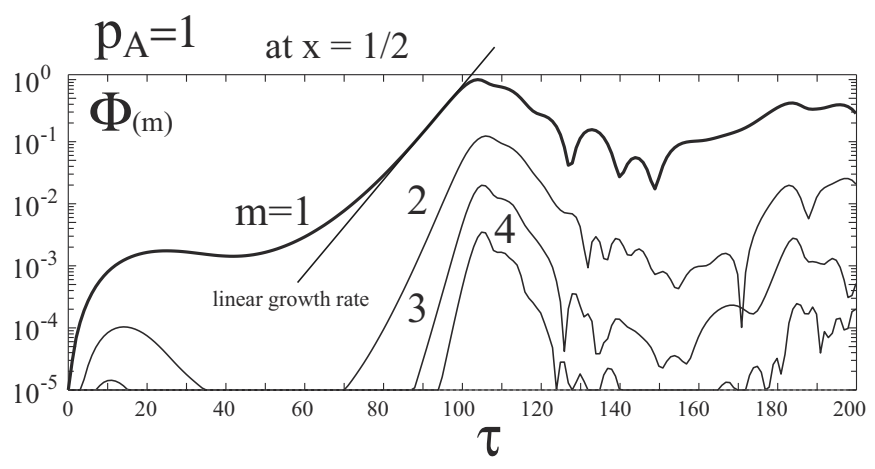

FIG. 5. Linear phase for $p_{A}=1$. chosen to be marginally stable to the flute modes, a large transport near the separatrix leads the system to an unstable state.

Figure 5 plots the time evolution of $\widetilde{\Phi}_{(m)}$ in the linear phase $(\tau \leq 200)$ observed at $x=1 / 2$. The $m=1$ Fourier amplitude $\widetilde{\Phi}_{(m)}$ grows in time, the growth rate of which agrees with the solid line in the figure. Solid line in Fig. 5 is the linear growth rate determined by the nonlocal linear analysis in Sec. IV, where the radial profiles of $D_{E}(x), T_{E}(x)$, and $\Phi_{0}(x)$ in Figs. 6(a) and 6(b) are used in the analysis. Here $D_{E}(x)$ and $T_{E}(x)$ in Figs. 6(a) and 6(b) are observed in the simulation at $\tau=80$. Figure 6(c) plots the radial profile of $m=1$ Fourier amplitude $\widetilde{\Phi}_{(m=1)}$ observed in the simulation at $\tau=80$, while Fig. 6(d) plots the radial profile of the eigenfunction of $\widetilde{\Phi}_{(m=1)}$ obtained by Eq. (19), where the eigenfunction was obtained in calculation of the linear growth rate in Fig. 5. Both radial profiles of $\widetilde{\Phi}_{(m=1)}$ and the eigenfunction agree well with each other. So the simulation results around $\tau=80$ are the linear growing phase. The contour plots of $w_{f}$, $\widetilde{\Phi}, D_{f}$, and $T_{f}$ in Fig. 7, which are observed at $\tau=80$, show that $m=1$ mode is dominant in all perturbations and those perturbations are localized around $x=1 / 2$. Here $\widetilde{\Phi}(x, \varphi)$ $\equiv \Phi(x, \varphi)-\Phi_{0}(x)$.

The long-time behavior of Fourier amplitudes $\widetilde{\Phi}_{(m)}$ is plotted in Fig. 8. The flute instability saturates at $\tau=100$ and after that the Fourier amplitudes $\widetilde{\Phi}_{(m)}$ repeat the growing up and down in time. The long-time behavior of these Fourier amplitudes can be understood in the following consideration.

Figures 9(a)-9(c) plot the time variations of $\Phi, \hat{D}$, and $\hat{T}$
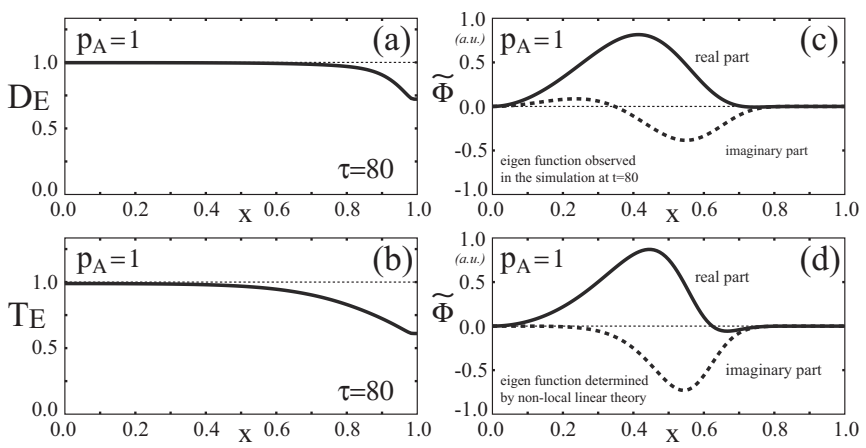

FIG. 6. Radial profiles of (a) $D_{E}(x)$, (b) $T_{E}(x)$ at $\tau=80$. Radial profiles of (c) the $m=1$ Fourier amplitude $\widetilde{\Phi}_{(m)}$ observed in the simulation at $\tau=80$, (d) the eigenfunction of $\widetilde{\Phi}_{(m=1)}$ obtained by the nonlocal linear analysis. 

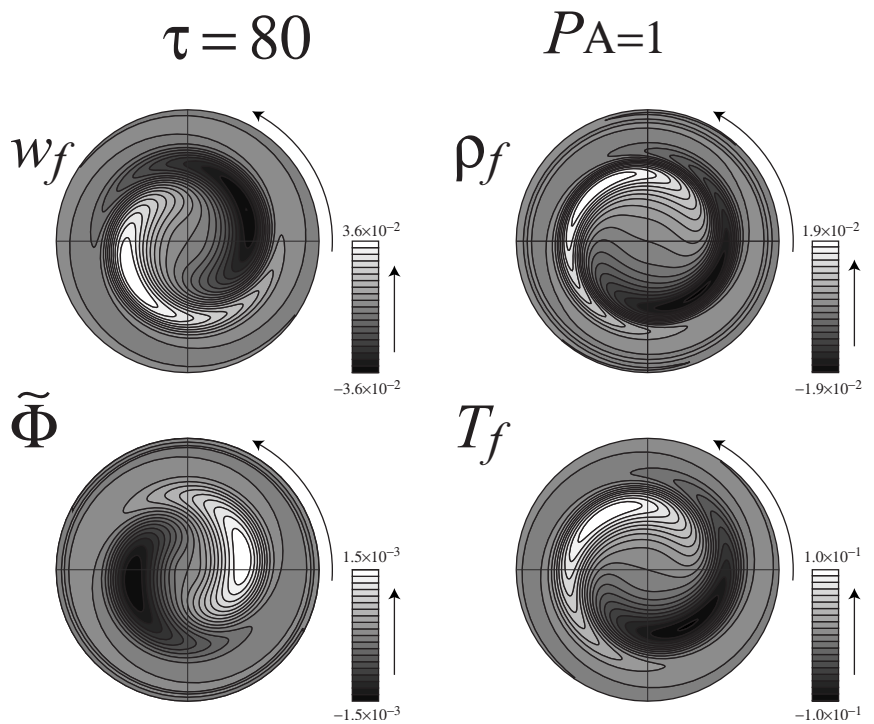

FIG. 7. Contour plots of $w_{f}, \widetilde{\Phi}, \rho_{f}$, and $T_{f}$ observed at $\tau=80$.

observed at $(x, \varphi)=(0,0)$ and $(1 / 3,0)$, while Fig. $9(\mathrm{~d})$ plots the time evolution of the Fourier amplitudes $\widetilde{\Phi}_{(m)}$. There are several features to be emphasized. The classical viscosity, resistivity, and thermal diffusivity are included in the basic Eqs. (11)-(13). It is seen that the magnitudes of $\hat{D}$ and $\hat{T}$ at $(x, \varphi)=(0,0)$ and $(1 / 3,0)$ decrease slowly due to the classical diffusion process in all time. There is a large drop of $\hat{D}(x=1 / 3, \varphi=0)$ and $\hat{T}(x=1 / 3, \varphi=0)$ accompanied with a rapid oscillation around $\tau=100$, which is just the same time as a flute instability saturates. The large drops accompanied with a rapid amplitude oscillation are observed repeatedly in time, which coincides with the time when the Fourier amplitude $\widetilde{\Phi}$ has a maximum in Fig. 9(d). The period of the rapid amplitude oscillation coincides with the period of the plasma azimuthal rotation.

Figure 10 plots the radial fluxes of density $\Gamma_{\rho}(x)$ and temperature $\Gamma_{T}(x)$, which are calculated by using the radial profiles of $\Phi, \hat{D}$, and $\hat{T}$ observed in the simulation at $\tau=100$. The radial fluxes $\Gamma_{\rho}(x)$ and $\Gamma_{T}(x)$ are divided into two terms

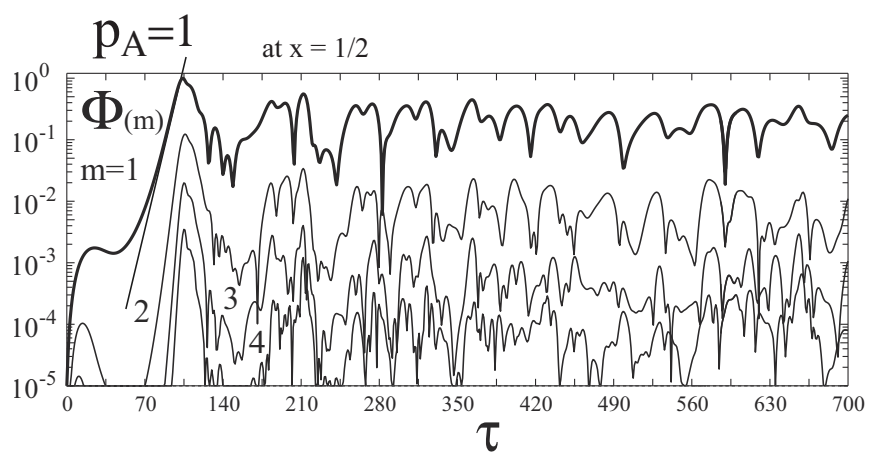

FIG. 8. Time variations of Fourier amplitude $\widetilde{\Phi}_{(m)}$ at $x=1 / 2$ in whole time.

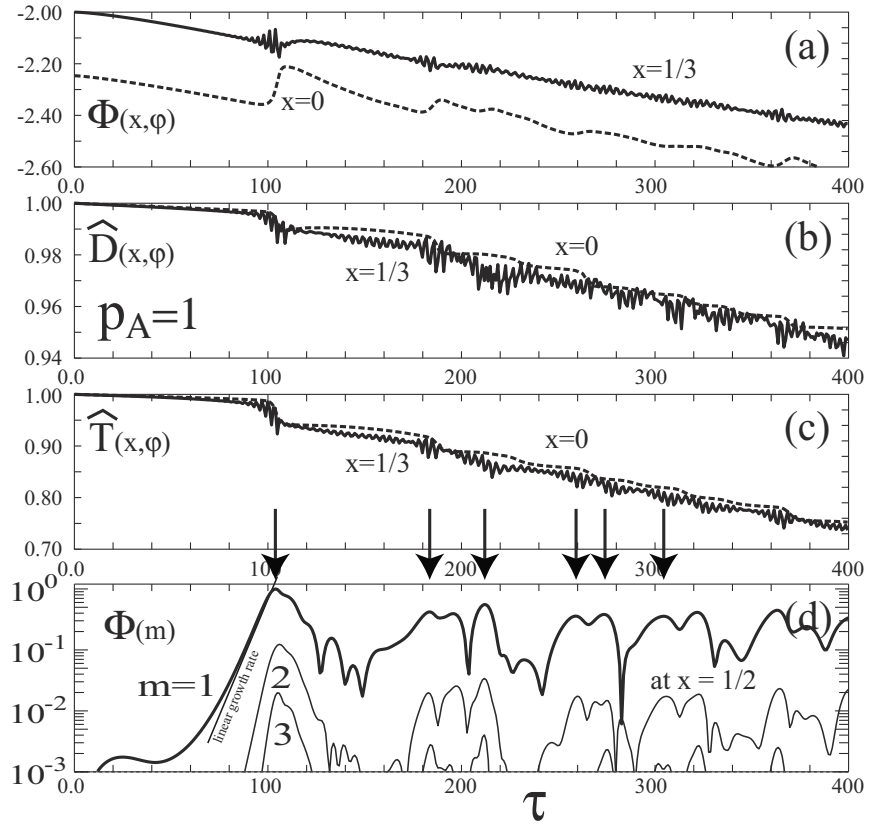

FIG. 9. Time variations of (a) $\Phi(x, \varphi)$, (b) $\hat{D}(x, \varphi)$, and (c) $\hat{T}(x, \varphi)$, all of which are observed at $(x, \varphi)=(0,0)$ (dashed line) and $(x, \varphi)=(1 / 3,0)$ (solid line), and (d) the Fourier amplitudes of $\widetilde{\Phi}_{(m)}$.

$$
\Gamma_{\rho}(x)=\Gamma_{\rho}^{\text {class }}(x)+\Gamma_{\rho}^{\text {flute }}(x), \quad \Gamma_{T}(x)=\Gamma_{T}^{\text {class }}(x)+\Gamma_{T}^{\text {flute }}(x) .
$$

Here $\Gamma_{\rho}^{\text {class }}$ and $\Gamma_{T}^{\text {class }}$ are the radial fluxes due to the classical transport, and $\Gamma_{\rho}^{\text {flute }}$ and $\Gamma_{T}^{\text {flute }}$ are the radial fluxes due to flute mode fluctuations, which are defined, respectively, as

$$
\begin{aligned}
& \Gamma_{\rho}^{\text {class }}=\frac{\epsilon^{2} C_{1}}{2} \frac{f_{1} D_{E}}{x T_{E}^{3 / 2}} \frac{\partial}{\partial x}\left(\frac{D_{E} T_{E}}{u^{5 / 3}}\right), \\
& \Gamma_{\rho}^{\text {flute }}=\frac{1}{2 \pi u} \int_{0}^{2 \pi} D_{f} \frac{\partial \tilde{\Phi}}{\partial \varphi} d \varphi, \\
& \Gamma_{T}^{\text {class }}=\frac{\epsilon^{2} C_{3}}{6} \frac{f_{1} D_{E}}{x u^{2 / 3} \sqrt{T_{E}}} \frac{\partial}{\partial x}\left(\frac{T_{E}}{u^{2 / 3}}\right),
\end{aligned}
$$
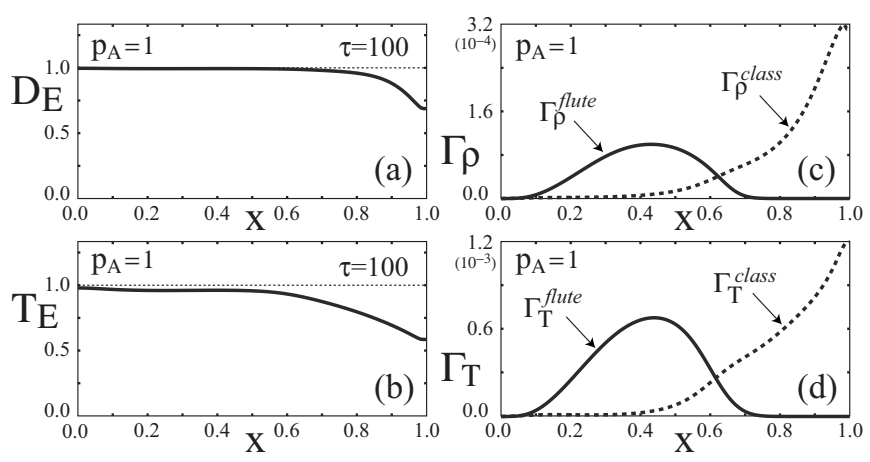

FIG. 10. Radial profiles of (a) $D_{E}(x)$ and (b) $T_{E}(x)$ at $\tau=100$. Radial fluxes of (c) density $\Gamma_{\rho}(x)$ and (d) temperature $\Gamma_{T}(x)$ measured at $\tau=100$. Solid lines in (c) and (d) are the anomalous diffusion and dashed lines are classical diffusion. 


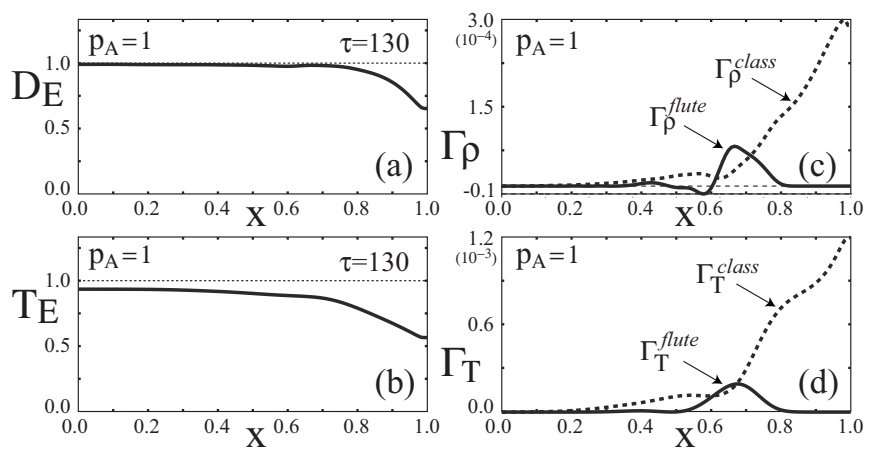

FIG. 11. (a) $D_{E}(x)$ and (b) $T_{E}(x)$ at $\tau=100$. (c) $\Gamma_{\rho}(x)$ and (d) $\Gamma_{T}(x)$ at $\tau=130$. Solid lines in (c) and (d) are the anomalous diffusion and dashed lines are classical diffusion.

$$
\Gamma_{T}^{\text {flute }}=\frac{1}{2 \pi u^{2 / 3}} \int_{0}^{2 \pi} T_{f} \frac{\partial \widetilde{\Phi}}{\partial \varphi} d \varphi .
$$

The classical radial fluxes $\Gamma_{\rho}^{\text {class }}$ and $\Gamma_{T}^{\text {class }}$ become large around $x \simeq 1$ in Figs. 10(c) and 10(d) because ion Larmor radius is large around the magnetic null. Therefore, there are sharp gradients in $D_{E}$ and $T_{E}$ around $x \simeq 1$ in Figs. 10(a) and 10(b). On the other hand, the radial fluxes $\Gamma_{\rho}^{\text {flute }}$ and $\Gamma_{T}^{\text {flute }}$ due to flute instabilities are found to be large around $x \simeq 0.4$ in Figs. 10(c) and 10(d) where the $m=1$ Fourier amplitude $\widetilde{\Phi}_{(m)}$ has a maximum in Fig. 6(c), which causes the large transports shown in Figs. 9(b) and 9(c).

Radial fluxes at $\tau=130$ are plotted in Fig. 11, when is in the decay phase of flute modes as shown in Fig. 5. The radial fluxes $\Gamma_{\rho}^{\text {flute }}$ and $\Gamma_{T}^{\text {flute }}$ are found to be smaller than the classical radial fluxes $\Gamma_{\rho}^{\text {class }}$ and $\Gamma_{T}^{\text {class }}$ in the core region. So the large transport due to flute mode fluctuations is not observed at $\tau \simeq 130$ in Figs. 9(b) and 9(c).

Figures 9-11 indicate that a maximum of Fourier amplitude $\widetilde{\Phi}$ is accompanied with a large radial transport. As a result of the large radial transport, the stable $D_{E}(x)$ and $T_{E}(x)$ are realized in the core region, which stabilizes the flute mode and makes its Fourier amplitude decrease. That is, the saturation mechanism of the flute instability at $\tau=100$ in Fig. 8 is that the radial profiles of $D_{E}(x)$ and $T_{E}(x)$ changed to satisfy the stable radial profile $D_{E}(x) T_{E}(x) \simeq$ const as a result of the large anomalous radial transport.

The large classical transport around $x \simeq 1$ makes the radial profiles of $D_{E}(x)$ and $T_{E}(x)$ unstable to the flute modes and the Fourier amplitude $\widetilde{\Phi}_{(m)}$ grows again. The resultant large diffusion by the flute instability forms the stable pressure profile and the Fourier amplitudes decays. This process is repeated and the radial transport is enhanced by the flute mode fluctuations.

\section{B. The case stable to a flute mode}

In this section the simulation is performed in the case of $p_{A}=20$, where a magnetic well exists in the region $x<0.45$ in Fig. 3. The purpose in this section is to make clear whether the flute mode is stabilized by a magnetic well or

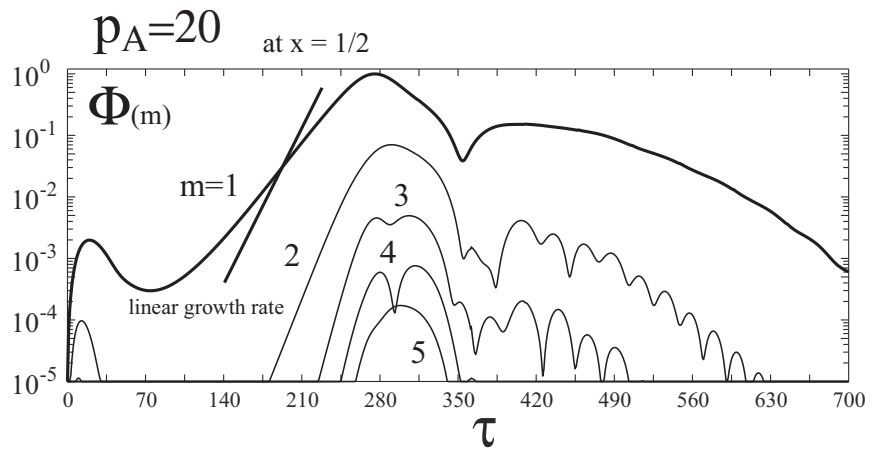

FIG. 12. Time evolutions of the Fourier amplitude $\widetilde{\Phi}_{(m)}$ in the linear phase for $p_{A}=20$.

not in a tandem mirror with a divertor mirror cell. The same boundary conditions and initial conditions as those in Sec. IV are adopted here.

Time evolutions of $\widetilde{\Phi}_{(m)}$ at $x=1 / 2$ are plotted in Fig. 12. The solid line in Fig. 12 is the linear growth rate obtained by the nonlocal linear analysis in Sec. IV, where the radial profiles of $D_{E}(x), T_{E}(x)$, and $\Phi_{0}(x)$ observed in the simulation at $\tau=180$ were used in the analysis. It is found that the growth rate observed in the simulation does not agree with the linear analysis, and all $m$ modes of $\widetilde{\Phi}_{(m)}$ decrease in the nonlinear saturation phase $(\tau>280)$.

Figure 13 shows the radial profiles of $m=1$ Fourier amplitude $\widetilde{\Phi}$, and the radial profiles of $D_{E}$ and $T_{E}$ observed at $\tau=180$, which were used to evaluate the linear growth rate in Fig. 12. Although $\partial\left(D_{E} T_{E}\right) / \partial x \neq 0$ in whole region, i.e., can be unstable to the flute modes, the radial profile $\widetilde{\Phi}_{(m=1)}(x)$ in Fig. 13(c) is different from the eigenfunction obtained by the nonlocal linear analysis. Because both growth rate and eigenfunction observed in the simulation are different from the linear analysis, the case of $p_{A}=20$ is understood to be not unstable to the flute modes.

Radial profiles of $D_{E}$ and $T_{E}$ at $\tau=320$ are shown in Fig. 14. In the region $x \leqq 0.45$, where $\partial U / \partial x<0$, the $D_{E}(x) T_{E}(x)$ has a Gaussian type of radial profile. The classical transport increases in $x$ just like Figs. 10 and 11, so the radial profiles of $D_{E}$ and $T_{E}$ have sharper gradients in $x \geqslant 0.5$. Note that $D_{E} \propto \rho U$ and $T_{E} \propto\left(T_{i}+T_{e}\right) U^{2 / 3}$ in Eq. (9).
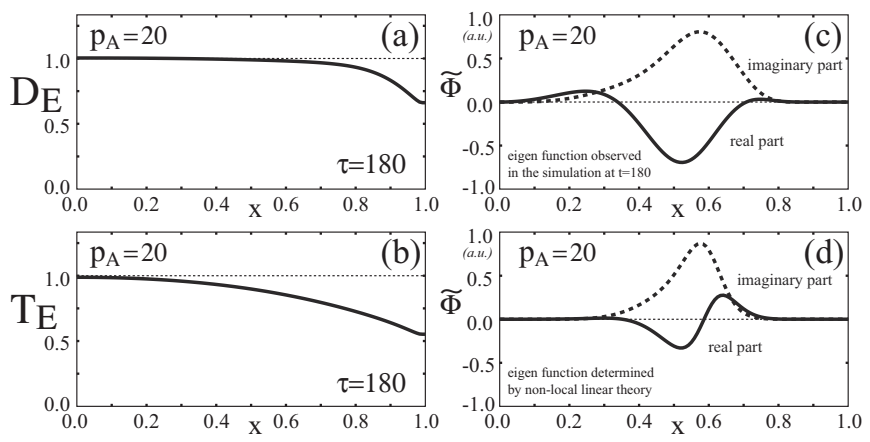

FIG. 13. Radial profiles of (a) $D_{E}(x)$, (b) $T_{E}(x)$, and (c) the $m=1$ Fourier amplitude $\widetilde{\Phi}_{(m)}$, all of which are observed in the simulation at $\tau=180$, and (d) the eigenfunction $\tilde{\Phi}_{(m=1)}$ obtained by the nonlocal linear analysis. 


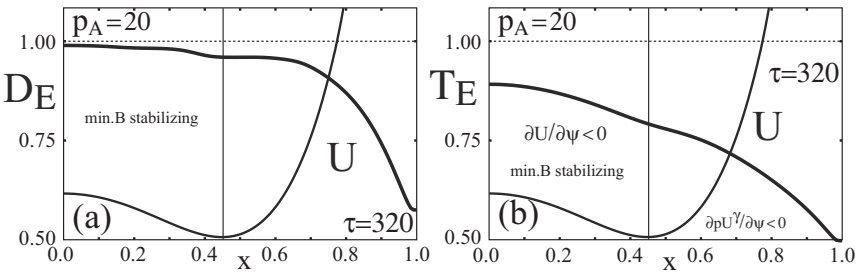

FIG. 14. Radial profiles of (a) $D_{E}(x)$ and (b) $T_{E}(x)$ observed at $\tau=320$.

\section{Various values of $p_{A}$ to a flute mode}

The simulations for various $p_{A} \mathrm{~s}$ are performed. The maximum amplitudes of $m=1$ Fourier component $\widetilde{\Phi}_{(m)}$ at $x=2 / 5$ are plotted in Fig. 15 . The maximum amplitude $\widetilde{\Phi}_{\max }$ of $\widetilde{\Phi}_{(m=1)}$, for example, is realized at $\tau=104$ in Fig. 8(d) and at $\tau=272$ in Fig. 12. The maximum amplitude $\widetilde{\Phi}_{\max }$ in the case of $p_{A}=20$ is one order magnitude smaller than the other case of $p_{A}$. The growth rates of $m=1$ Fourier amplitude $\widetilde{\Phi}_{(m)}$ in the linear growing phase are plotted in Fig. 16 as a function of $p_{A}$. Here solid triangles are the linear growth rates observed in the simulation where $\tau$ 's in the figure are the time measured the growth rate of $\widetilde{\Phi}_{(m=1)}$, while solid circles are the linear growth rates determined by the nonlocal linear analysis. The agreement between simulation and linear analysis is good for $p_{A} \leq 12$. There is not a simulation result at $p_{A}=20$ in Fig. 16 because flute modes are not unstable in the simulation.

A magnetic well exists for $p_{A} \gtrsim 12$ as shown in Fig. 3. The effects of the magnetic well to the flute modes are clearly seen in Figs. 15 and 16 in a sense that the linear growth rate and the maximum amplitude $\widetilde{\Phi}_{\max }$ are made lower by the magnetic well. If the plasma has a slim radial pressure profile of $\partial p U^{\gamma} / \partial \psi<0$, the divertor mirror does not stabilize the flute modes as mentioned in Sec. II. Because the divertor mirror has a bad magnetic field line curvature near axis, the magnetic well (region of the good curvature) in the anchor minimum-B mirror cell can stabilize the flute modes. ${ }^{17,25,26}$ The flute modes are stabilized completely by the magnetic well for $p_{A} \gtrsim 20$.

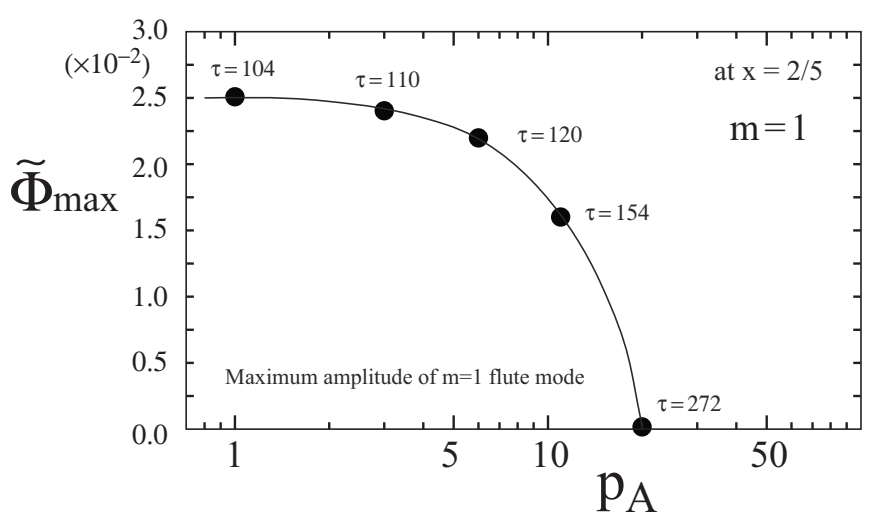

FIG. 15. The maximum $m=1$ Fourier amplitude $\widetilde{\Phi}_{(m)}$ observed in the simulations vs $p_{A}$.

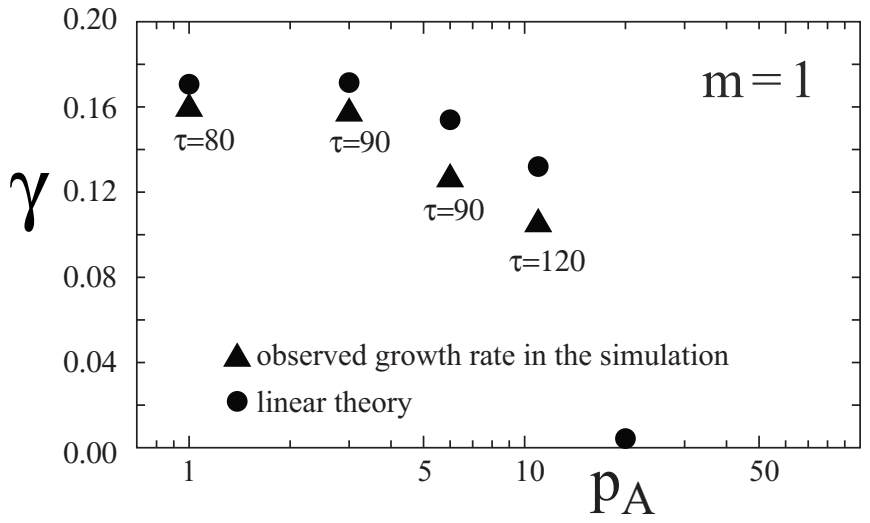

FIG. 16. Linear growth rates: analytical calculation (solid circles) and observed in the simulation (solid triangles).

\section{SUMMARY}

The simulations on flute modes were performed in order to research the flute instability, the associated radial transport, and the effect of a magnetic well to the flute instability. The linear growth rates and the radial profiles of the eigenfunction $\widetilde{\Phi}_{(m=1)}$ in the linear phase agree well between the simulation and nonlocal linear analysis. It was found that the flute instability entered a nonlinear saturation phase by changing the unstable radial profile $D_{E}(x) T_{E}(x)$, which was caused by the large classical transport around $x \simeq 1$, to the stable radial profile resulting from the flute instability after a linear phase. In the saturation phase the flute modes repeat the grow up and down of its Fourier amplitudes causing the anomalous radial transport intermittently. The flute instability, however, is not so strong to evacuate the plasma out of separatrix fast. The perturbed potential $\widetilde{\Phi}$ caused by the flute instabilities has the same amplitude along the magnetic field line because of $k_{\|}=0$, thus the radial transports are the same in each mirror cell.

The magnetic well $(\partial U / \partial \psi<0)$ near axis $(\psi \simeq 0)$ stabilizes the flute modes in the long thin magnetic field. On the other hand, the divertor magnetic field has a character $\partial U / \partial \psi \rightarrow+\infty$ at separatrix. Thus the tandem mirror with both a divertor mirror and a minimum-B mirror has the character that $\partial U / \partial \psi<0$ near axis but $\partial U / \partial \psi \gg 1$ near separatrix. It was found that the magnetic well was not able to stabilize the flute modes in such a tandem mirror if the magnetic well was shallow. That is, the divertor mirror weakens the ability of minimum-B mirror to stabilize the flute modes.

Here we comment on the condition in which the divertor cell is stabilizing the flute modes because the experiments reported the stabilizing effects. ${ }^{22-24}$ The experiments realize the steady state operation where the plasma radial loss is balanced with the plasma production. The flute modes can be stabilized by the divertor as mentioned in Sec. II if the radial pressure profile is fat. The simulation, however, starts at the initial condition but without the plasma source. The radial loss through the x-point, therefore, makes the pressure radial profile slim, which makes the flute modes unstable. 


\section{ACKNOWLEDGMENTS}

The authors wish to acknowledge the valuable discussions with Dr. V. P. Pastukhov.

${ }^{1}$ G. I. Dimov, V. V. Zakaidakov, and M. E. Kishinevskij, Sov. J. Plasma Phys. 2, 326 (1976).

${ }^{2}$ T. K. Fowler and B. G. Logan, Comments Plasma Phys. Controlled Fusion 2, 167 (1977)

${ }^{3}$ M. Inutake, T. Cho, M. Ichimura, K. Ishii, A. Itakura, I. Katanuma, Y. Kiwamoto, Y. Kusama, A. Mase, S. Miyoshi, Y. Nakashima, T. Saito, A. Sakasai, I. Wakaida, N. Yamaguchi, and K. Yatsu, Phys. Rev. Lett. 55, 939 (1985).

${ }^{4}$ N. Oyabu, T. Watanabe, H. Akao, T. Ono, T. Kawamura, K. Yamazaki, K. Akaishi, N. Inoue, A. Komori, Y. Kubota, N. Noda, A. Sagawa, H. Suzuki, O. Motojima, M. Fujiwara and A. Ilyoshi, Nucl. Fusion 34, 387 (1994).

${ }^{5}$ N. Ohyabu, A. Komori, H. Suzuki, T. Morisaki, S. Masuzaki, H. Funaba, N. Noda, Y. Makamura, A. Sagara, N. Inoue, R. Sakamoto, S. Inagaki, S. Morita, Y. Takeiri, T. Watanabe, O. Motojima, M. Fujiwara, and A. Iiyoshi, J. Nucl. Mater. 266-269, 302 (1999).

${ }^{6}$ N. Asakura, H. Kawashima, K. Shimizu, S. Sakurai, T. Fujita, H. Takenaga, T. Nakano, H. Kubo, S. Higashijima, T. Hayashi, Y. Kamada, S. Ide, M. Kikuchi, Y. Takase, Y. Ueda, N. Ohno, M. Sakamoto, O. Gruber, A. Kallenbach, A. Grosman, and M. Lipa, Proceedings of the 34th EPS Conference on Plasma Physics, Warsaw, 2-6 July 2007, Vol. 31F, p. 1.051 .

${ }^{7}$ G. Janeschitz, R. Tivey, A. Antipenkov, V. Barabash, S. Chiocchio, G. Federici, H. Heidl, C. Ibbott, and E. Martin, Fusion Eng. Des. 49-50, 107 (2000).

${ }^{8}$ I. Katanuma, K. Yagi, Y. Nakashima, M. Ichimura, and T. Imai, Phys. Plasmas 17, 032303 (2010).

${ }^{9}$ I. Katanuma, Y. Kiwamoto, K. Ishii, K. Yatsu, and S. Miyoshi, Phys. Fluids B 1, 1459 (1989).

${ }^{10}$ D. D. Ryutov and G. V. Stupakov, Fiz. Plazmy 4, 501 (1978); Sov. J. Plasma Phys. 4, 278 (1978).

${ }^{11}$ D. D. Ryutov and G. V. Stupakov, Dokl. Akad. Nauk SSSR 240, 1086 (1978); Sov. Phys. Dokl. 23, 412 (1978).

${ }^{12}$ R. H. Cohen, Nucl. Fusion 19, 1579 (1979).
${ }^{13}$ I. Katanuma, Y. Kiwamoto, S. Adachi, M. Inutake, K. Ishii, K. Yatsu, K. Sawada, and S. Miyoshi, Nucl. Fusion 27, 2041 (1987).

${ }^{14}$ J. A. Wesson, D. J. Ward, and M. N. Rosenbluth, Nucl. Fusion 30, 1011 (1990).

${ }^{15}$ M. Wakatani and T. Abe, J. Phys. Soc. Jpn. 51, 1969 (1982).

${ }^{16}$ D. N. Hill, J. Nucl. Mater. 241-243, 182 (1997).

${ }^{17}$ I. Katanuma, Y. Tatematsu, K. Ishii, T. Tamano, and K. Yatsu, J. Phys. Soc. Jpn. 69, 3244 (2000).

${ }^{18}$ K. Miyamoto, Plasma Physics for Nuclear Fusion (MIT Press, Cambridge, 1979).

${ }^{19}$ B. Lane, R. S. Post, and J. Kesner, Nucl. Fusion 27, 277 (1987).

${ }^{20}$ V. P. Pastukhov and A. Yu. Sokolov, Fiz. Plasmy 17, 1043 (1991); Sov. J. Plasma Phys. 17, 603 (1991).

${ }^{21}$ Y. Sasagawa, I. Katanuma, Y. Mizoguchi, T. Cho, and V. P. Pastukhov, Phys. Plasmas 13, 122506 (2006)

${ }^{22}$ J. A. Casey, B. G. Lane, J. H. Irby, K. L. Brau, S. N. Golovato, W. C. Guss, J. Kesner, P. S. Post, E. Sevillano, and J. Zielinski, Phys. Fluids 31, 2009 (1988).

${ }^{23}$ Y. Yasaka, M. Takano, and H. Takeno, Trans. Fusion Technol. 39, 350 (2001).

${ }^{24}$ A. C. England, D. K. Lee, S. G. Lee, M. Kwon, S. W. Yoon, Y. Yasaka, N. Sugimoto, I. Katanuma, K. Yashiro, and T. Imai, Nucl. Fusion 49, 125008 (2009).

${ }^{25}$ T. B. Kaiser and L. D. Pearlstein, Phys. Fluids 26, 3053 (1983).

${ }^{26}$ T. B. Kaiser, W. M. Nevins, and L. D. Pearlstein, Phys. Fluids 26, 351 (1983).

${ }^{27}$ R. F. Post, Trans. Fusion Technol. 39, 25 (2001).

${ }^{28}$ D. D. Ryutov, Physics of Mirrors, Reversed Field Pinches, and Compact Tori: Proceedings of the Course and Workshop (Villa Monastero, Varenna, 1987), pp. 791-815.

${ }^{29}$ M. N. Rosenbluth and C. L. Longmire, Ann. Phys. 1, 120 (1957).

${ }^{30}$ V. P. Pastukhov, Fiz. Plazmy 31, 628 (2005); Plasma Phys. Rep. 31, 577 (2005).

${ }^{31}$ I. Katanuma, V. P. Pastukhov, T. Imai, M. Ichimura, T. Kariya, Y. Nakashima, H. Hojo, R. Minami, Y. Yamaguchi, M. Yoshikawa, H. Akao T. Watanabe, and W. Horton, J. Plasma Fusion Res. 84, 279 (2008).

${ }^{32}$ S. I. Braginskii, in Reviews of Plasma Physics, edited by A. M. A. Leontovich (Consultants Bureau, New York, 1965), Vol. 1. 